\title{
ADVANCED APPROACH FOR REDUCING PAPR IN OFDM SYSTEMS TO MINIMIZE INTERFERENCE EFFECT
}

\author{
Nutan Kumari ${ }^{1}$, Simarpreet Kaur ${ }^{2}$ \\ ${ }^{I}$ M.tech Student,Deptt. of Electronics, Baba Banda Singh Bahadur Engineering College, Fatehgarh Sahib, Punjab, \\ India \\ ${ }^{2}$ Assistant Professor, Deptt. of Electronics, Baba Banda Singh Bahadur Engineering College, Fatehgarh Sahib, \\ Punjab, India
}

\begin{abstract}
Orthogonal Frequency Division Multiplexing is a popular modulation scheme and finds its use in wireless communication and mostly drawing attention towards $4 G$ mobile communication. Wireless Technology is cardinal as it offers high data rate transmission, which is needed for efficacious communication. The main features of OFDM technique are that it can easily acclimate to severe channel conditions without complex time-domain equalization and is vigorous against Intersymbol Interference (ISI) and fading caused by multipath propagation. But the OFDM system faces the problem of PAPR i.e. Peak to Average Power Ratio. This PAPR effect is tried to be reduced using a combined technique which makes use of clipping and filtering technique along with companding technique. The simulation results show us that the PAPR value reduces to 4.41dB with a value of 128 subcarriers. Earlier used approaches for PAPR reduction were SLM, PTS, Tone reservation etc. The proposed approach shows that PAPR effect is tried to be reduced to a great extent.
\end{abstract}

Key Words: OFDM, PAPR, BER,FFT, IFFT etc.

\section{INTRODUCTION}

In recent years, wireless technology has gained an immense popularity and is in the mainstream because of high data rate transmission. Basically, The OFDM System works on the principle of division of available spectrum into various orthogonal sub channels so that each narrowband sub channel experiences nearly flat fading. The OFDM Systems can manage the frequency selective fading channel. The OFDM system finds many applications in $4 \mathrm{G}$ wireless communication systems, digital -audio broadcasting, digital-video broadcasting and wireless LAN networks[9].The recent use of OFDM System is to reduce the interference effect so that data transmission over multipath channels can take place efficiently. The features of OFDM System comprises of efficient system capacity, highly improved bandwidth efficiency,high spectral efficiency[7],[9] and handy implementation. But the major drawback that the system faces is the high value of PAPR i.e. peak to average power ratio. The elevated value of PAPR will expand the system complexity and thus will cause the in-band radiation and out of band distortion. Diversified techniques have been proposed to reduce the PAPR effect. and the PAPR Reduction techniques can be broadly classified into two categories i.e. signal distortion and signal scrambling techniques. These techniques include selective mapping (SLM), Partial transmit Sequence(PTS), Tone reservation, clipping and filtering and companding technique. In this paper, a new approach which makes use of clipping and filtering along with companding technique is utilized to reduce PAPR effect is proposed here. The simulation results show that the proposed design has better performance compared with the individual techniques. The assembling of this paper is as follows:section 2 introduces the OFDM system model ,section 3 explains the PAPR in OFDM system, section $4 \& 5$ give brief idea about clipping-filtering and companding technique, section 6 presents the proposed scheme which uses clipping and filtering along with companding technique, section 7 gives us the simulations and results and section 8 sums up with the conclusion and future work.

\section{OFDM SYSTEM MODEL}

An OFDM Carrier signal comprises of orthogonal subcarriers which carries baseband data. The baseband data on individual sub-carrier is being modulated using either Quadrature amplitude modulation or Phase shift keying.The OFDM Systems are taken into practice using FFT and IFFT i.e. (Fast Fourier transform ) and (Inverse fast Fourier transform) [6].The OFDM system divides the available frequency spectrum into diversified orthogonal sub-carrier channels and there are streams which carry higher bit rate.

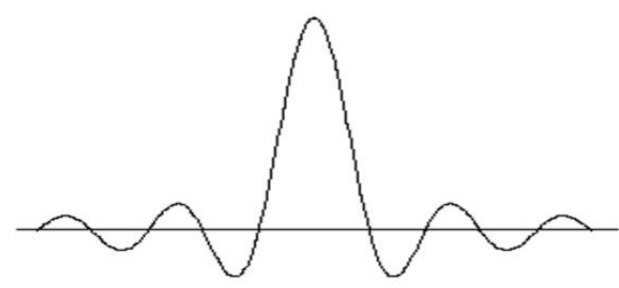

Fig 2.1 It depicts the spectrum of an OFDM Sub channel during single bit[8]. 


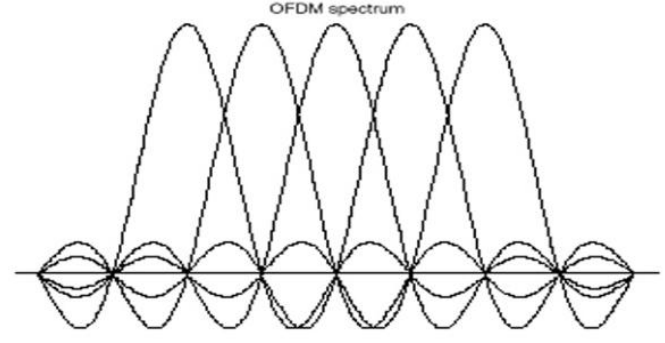

Fig 2.2 shows OFDM Spectrum[8].
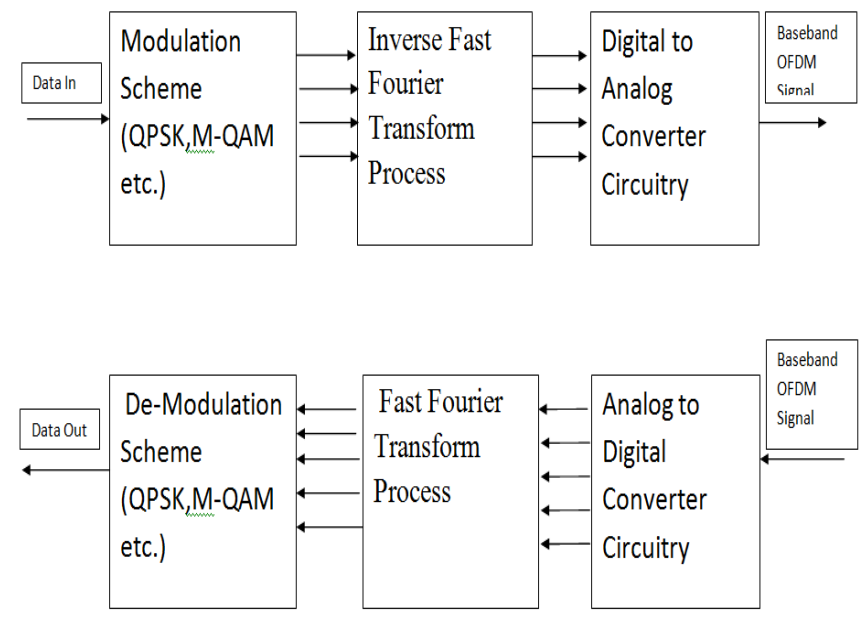

Fig 2.3 Block Diagram of OFDM Transmitter and Receiver

At the transmitter end a stream of $\mathrm{n}$ bits is transmitted. Then a modulation scheme is applied on the sub-carriers. The modulation scheme can differ depending on the sub-carriers. An IFFT is applied to $\mathrm{n}$ complex numbers i.e. Inverse fast Fourier transform. The stream is applied to serial to parallel converter [9]. The received signal is applied to the parallel to serial converter and the Fast Fourier Transform operation is applied to the n complex numbers to produce the symbol. The bits are obtained through demodulation techniques and hence the output signal is obtained in the OFDM system, the streams are recombined into a serial stream which is an estimate of original binary stream at the transmitter.

\section{PAPR IN OFDM SYSTEM}

The upraised value of Peak to Average Power Ratio is one of the major stumbling blocks of the OFDM modulation.The OFDM Signal is fabricated using a large number of independent modulated sub-carriers. The OFDM Signal contributes to a upraised value of PAPR when these independent modulated sub-carriers gets added up orderly[4]. when $\mathrm{n}$ signals are added with same phase they produce a peak power that is $\mathrm{N}$ times the average power of the signal. So OFDM Signal exhibits a high PAPR, which is sensitive to non-linearity of high power amplifier. Whereas, on the other hand,low PAPR makes the transmit power amplifier works efectively.The non-linearity in the signal gives rise to many obstacles such as Inter-carrier Interference(ICI), Intersymbol interference(ISI).

The probabilty that the PAPR of a certain block has exceeded the given threshold is measured using complementary CDF [CCDF].PAPR is the emerging issue in the field of the OFDM systems and a lot of attention has been grabbed by the wireless communication industry. Where $x(t)$ is represented by the following equation[1]:-

$\frac{1}{\sqrt{N}} \sum_{k=0}^{N-1} X_{K} e^{j 2 \pi f_{n} t}, 0<t<T$

where $X_{K}=\left\{X_{0}, X_{1} \quad X_{n}\right\}$ represents OFDM signal Blocks, $f_{n}=\left\{f_{0} f_{1}, \ldots \ldots . f_{N-1}\right\}$ represents $\mathrm{N}$ orthogonal subcarriers.

PAPR is stated as follows[7]:-

$$
\operatorname{PAPR}(\mathrm{x})=\frac{\max |\mathrm{x}(\mathrm{t})|^{2}}{\mathrm{E}\left[|\mathrm{x}(\mathrm{t})|^{2}\right]}
$$

Where E[.] depicts Expectation operator.

\section{CLIPPING \&FILTERING TECHNIQUE}

Clipping and Filtering is an elementary technique used for PAPR Reduction in OFDM systems. The operation of the technique is simplified by its name itself. Basically it clips the part of the signal when it cuts across a certain selected average area. The main feature of the clipping technique is that it is capable in removing components of the broadened spectrum. The limitations of this technique can be specified as introduction of distortion power, clipping noise and interference[3]. It causes in-band distortion which further accounts degradation in BER and spectral efficiency. The clipping and filtering approach helps in clipping the extended signal levels that are not allowed to cut across a certain level[3].On the other hand, filtering provides decrease in the spectrum growth. So to avoid peak regrowth problem, clipping and filtering technique is made in use[3]. This technique requires numerous iterations to clip the signal to avoid peak regrowth problem. But in recent years several algorithms have been proposed which employs the use of single iterative scheme to minimize the interference effect.[5],[1]. The clipping signal is classified as[2] :-

$$
C(X)= \begin{cases}x_{x} & |x| \leq A \\ A_{x} & |x| \geq A\end{cases}
$$

Where $A$ is a positive real number which represents clip level.

\section{COMPANDING TECHNIQUE}

The term companding is formed by the use of two words i.e. compression and expansion. So companding is a reduction technique used for reducing the PAPR effect in OFDM signals. The companding transform in which compression and expression is done makes use of IFFT and FFT process. With the help of this technique, large signals are allowed to be transmitted over ranges which have smaller range capability. The system implementation complexity is reduced and the gain of the signal is raised and PAPR effect is reduced. The compression is done at transmitter end after the IFFT process and expansion is done at the receiver end prior to FFT process. 


\section{PROPOSED SCHEME}

In OFDM technique one of the major problems is of PAPR, as it increases the system complexity and also reduces the gain of the signal, so there is need to reduce the effect of the PAPR in the signal. The PAPR decrease strategies differ as per the needs of the system and depend on different elements. PAPR reducing capacity, increment in power in transmit signal, loss in information rate, unpredictability of processing and increment in the bit-error rate at the receiver end are different variables which are considered before adopting a PAPR decrease strategy of the system. By studying pervious PAPR reduction techniques a new approach is proposed in this paper. Earlier Clipping and filtering was done, which were not able to reduce the effect of PAPR to that extent.As clipping can only remove a part of signal and filtration made signal smooth.

A new approach is made on the basis of combination of methodology. Previously clipping and filtration were used. In this along with clipping and filtration, companding of signal is also done. As companding allows signals with a large dynamic range to be transmitted over facilities that have a smaller dynamic range capability This approach is quite useful as it not only reduce the PAPR effect but also decreases the complexity in attenuation ,moreover the gain of signal is raised and the PAPR effect is reduce to great extent .

\section{SIMULATION RESULTS}

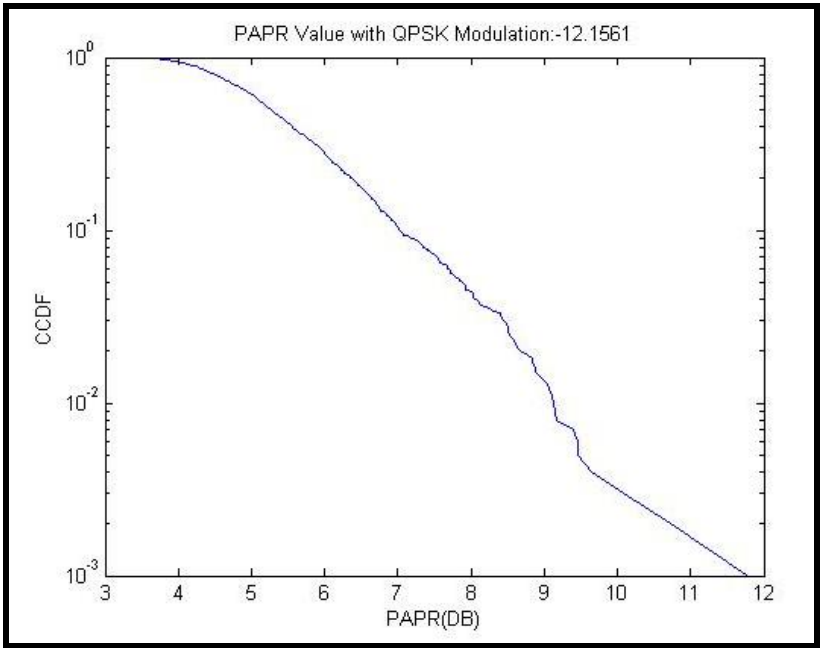

Fig 7.1 CCDF Vs PAPR Plot for Original OFDM Signal[1].

Figure 7.1 shows the CCDF Vs Plot before applying any PAPR reduction Technique.i.e. original OFDM Signal with PAPR value of approx. $12 \mathrm{~dB}$

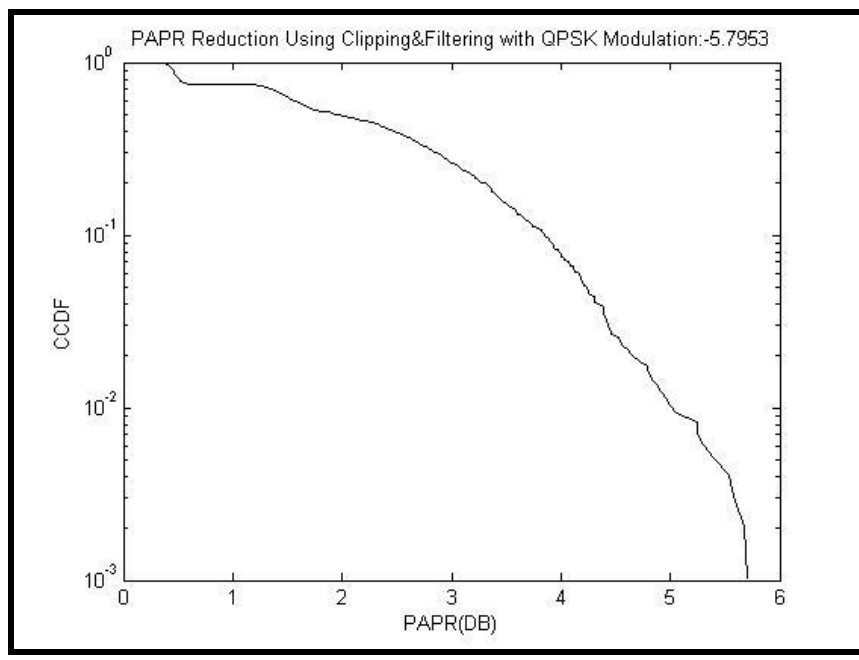

Fig 7.2 CCDF Vs PAPR Plot using clipping and filtering[1].

Figure 7.2 shows that the PAPR value gets reduced to $\sim 6 \mathrm{~dB}$ after the Papr Reduction technique i.e. clipping and filtering technique is applied to the OFDM signal.

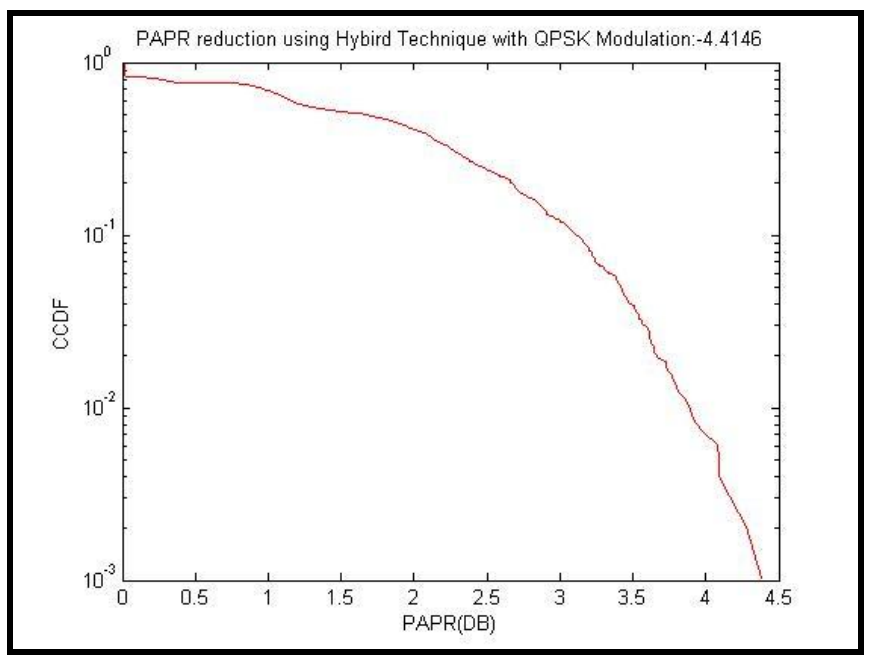

Fig 7.3 CCDF Vs PAPR Plot using advanced approach,i.e clipping and filtering along with companding technique.

Figure7.3 shows that the PAPR Value is reduced to 4.41decibels after the implemetation of advanced apporach i.e.clipping and filtering along with companding technique, which allows to reduce the effect of high peak to average power ratio to a great extent. 


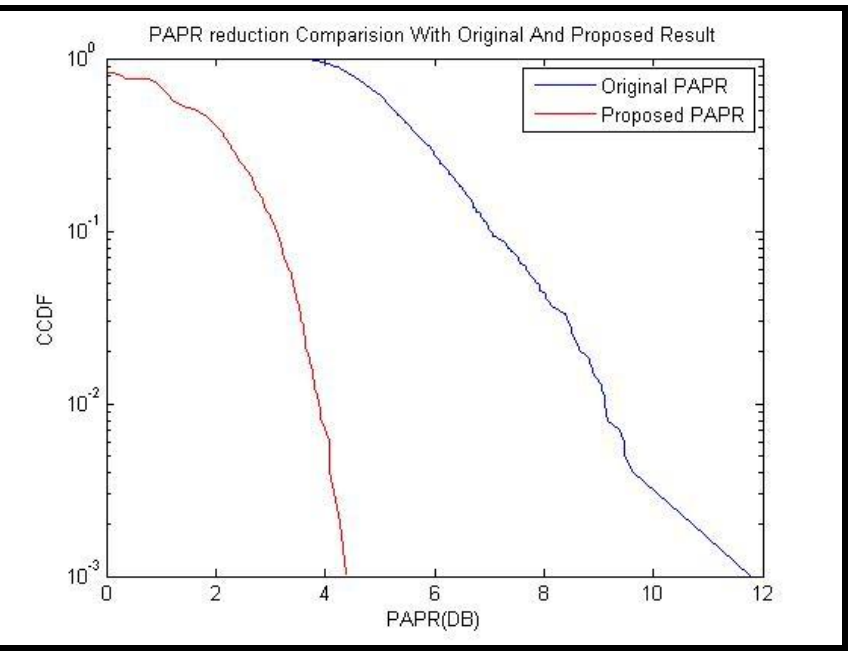

Fig 7.4 CCDF Vs PAPR Plot Comparison of Original OFDM Signal \&Proposed Result.

Figure7.4 depicts the comparison of the original PAPR value with the proposed PAPR value. The graph shows that original OFDM signal had the PAPR value of $12 \mathrm{~dB}$, but with the help of proposed technique,the PAPR value is tried to reduce to $4.41 \mathrm{~dB}$.

\section{CONCLUSIONS AND FUTURE WORK}

In this paper, an advanced approach is implemented which makes use of clipping and filtering along with companding technique to reduce the PAPR effect in OFDM systems. This new combined approach accomplishes a $4.41 \mathrm{~dB}$ PAPR reduction. This approach is quiet useful as it not only reduces the PAPR effect but also decreases the complexity in attenuation, moreover, the gain of signal is raised and the PAPR effect is tried to reduced to a great extent. For further work in PAPR reduction, the system can be used with Artificial Intelligence Systems and fuzzy systems, where the system will be trained and it will automatically reduce the PAPR Effect.

\section{REFERENCES}

[1]. Arvind Chakrapani,V. Palanisamy," A Novel Clipping and Filtering Algorithm Based on Noise cancellation for PAPR Reduction in OFDM Systems ,Springer,September 2014.

[2]. Gurtej Singh Toor, Harjinder Singh, Amandeep singh Bhandari, 'REVIEW PAPER ON PAPR REDUCTION TECHNIQUES IN OFDM SYSTEM', International Journal For Technological Research in Engineering,Vol 1,Issue 8,April 2014

[3]. Md. Ibrahim Abdullah, Md. Zulfiker Mahmud, Md. Shamim Hossain, Md. Nurul Islam, "Comparative Study of PAPR Reduction Techniques in OFDM", ARPN Journal of Systems and Softwares, Vol 1,NO.8,November 2011

[4]. Dhanvi Gupta, Divya Dhawan International Journal of Computer Applications ,Volume 115 - No. 15, "A Study of Various PAPR Reduction Techniques for Optical OFDM Systems”, April 2015
[5]. Heena Bhatti,Kritika Ramesh," A new single iterative clipping and filtering technique to reduce PAPR,(IOSR-JECE)Journal of electronics and communication Engineering, ,p-ISSN:2278-8735, PP $77-81$

[6]. Isha Arora,Mohit Kumar Singh,"Reduction of PAPR in OFDM Signal using Hybrid Clipping- $\mu$ Law companding technique, International Journal of Electrical Electronics \&Computer Science Engineering,Volume 1,Issue 6,December 2014

[7]. Arun Gangwar,Manushree Bhardwaj,"An overview:Peak to Average Power ratio in OFDM System \& its Effect, International Journal of communication and computer Technologies, Volume 1-No.2,Issue 2,September 2012.

[8]. www.wirelesscommunication.nl

[9]. www.wikipedia.org

\section{BIOGRAPHIES}

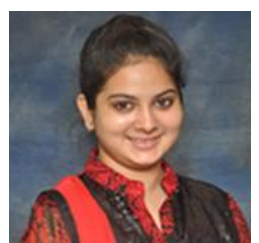

Nutan Kumari is pursuing M.Tech in Electronics and Comm.Engg. from Baba Banda Singh Bahadur Engineering College,Fatehgarh Sahib.Her topic of research is PAPR Reduction in OFDM Systems using advanced approach i.e. clipping and filtering along with companding technique to minimize interference effect.

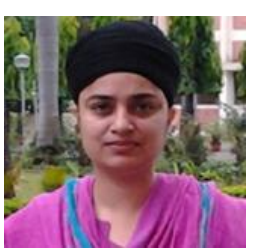

Prof.Simarpreet Kaur is Assistant Professor at Baba Banda Singh Bahadur Engineering College, Fatehgarh Sahib. Her areas of interest are Wireless and Sensor Networks, Image Processing and Optical Networks. 